\title{
Gene expression patterns of invertase gene families and modulation of the inhibitor gene in tomato sucrose metabolism
}

\author{
Y.L. Zhang, A.H. Zhang and J. Jiang \\ Key Laboratory of Protected Horticulture, Ministry of Education, \\ College of Horticulture, Shenyang Agricultural University, \\ Shenyang, China \\ Corresponding author: J. Jiang \\ E-mail: jj_syau@hotmail.com \\ Genet. Mol. Res. 12 (3): 3412-3420 (2013) \\ Received July 3, 2012 \\ Accepted September 28, 2012 \\ Published January 24, 2013 \\ DOI http://dx.doi.org/10.4238/2013.January.24.1
}

\begin{abstract}
Patterns of gene expression in the different types of sucrose metabolism in the tomato are highly variable and heritable. This genetic variation causes considerable functional differences. We examined the patterns of expression of invertase (Inv) gene families and an invertase inhibitor (INH) gene involved in elongating roots, hypocotyls, and fruit of the tomato (Lycopersicon esculentum cv. Micro-Tom and L. chmielewskii) through a real-time quantitative PCR analysis. We found that the Lin6 gene plays an important role in the vegetative growth stage. $\operatorname{Lin} 5$ and $\operatorname{Lin} 7$ did not express in Micro-Tom, but did express in L. chmielewskii. Overall relative expression levels of sucrose Inv gene families were significantly lower in L. chmielewskii during the reproductive growth stage than in Micro-Tom, being up to hundreds of times lower. It was not expressed in the dissepiment in L. chmielewskii. We suggest that differences in sucrose accumulation in tomato fruit is mainly due to differentially expressed invertase gene families at the later fruit growth stages.
\end{abstract}

Key words: Tomato; Invertase gene families; INH; RT-PCR 


\section{INTRODUCTION}

Tomatoes are widely cultivated around the world and crop yield and quality directly influence consumption. Sugar accumulation is the key to fruit quality, and sucrose metabolism is the important link in the accumulation of sugar. Invertase is the key enzyme in sucrose metabolism, participating in sucrose catabolism, reducing sucrose content, and has an important physiological role in vivo. Acid invertase (AI) is the most important enzyme in sucrose metabolism in the fruit. AI plays a major role in regulation of phloem sugar unloading, controlling the sugar composition in storage organs, influencing the response to biotic and abiotic stresses, affecting early plant growth and signal transduction, etc. AI is expressed in 2 forms: vacuole invertase (VI) and cell wall-bound invertase (CWI). In tomato, VI is encoded by TIVI; CWIs are encoded by a gene family including $\operatorname{Lin} 5, \operatorname{Lin} 6, \operatorname{Lin} 7$, and $\operatorname{Lin} 8$ (Godt and Roitsch, 1997). The Lin genes are located on the ninth and tenth chromosomes. An endogenous protein called an invertase inhibitor suppresses invertase activity in potato (Schwimmer et al., 1961). It also inhibits invertase activity in tomato (Jin et al., 2009).

Research on apple (Malus pumila Mill.), melon (Cucumis melo Linn.), tomato (Lycopersicon esculentum Mill.), and other crops has revealed the close relationship between enzyme activity in sucrose metabolism and fruit sugar accumulation (Beürter, 1985; Hubbard et al., 1989; Jiang and Li, 2005). This has laid the foundation for understanding of fruit sugar accumulation; however, many studies on sugar content during different periods of fruit development have not addressed fruit sugar composition and content in different parts of the fruit. Moreover, changes in the activity of enzymes related to each step of sugar metabolism are rarely described. Invertase is a key enzyme in sucrose metabolism and is important for sugar accumulation and composition in the fruit; it also affects plant growth and development. Invertase activity varies in different stages and organs of plant growth and development; thus, invertase (Inv) gene expression varies in time and space, with specific expression characteristics in each stage of development and tissue organ (Masao et al., 1991; Hedley et al., 1994). Understanding the Inv genes and invertase inhibitor will help define the underlying mechanisms of plant fertility and quality. The molecular mechanisms of sucrose metabolism, accumulation, and physiological function may be clarified by understanding the expression patterns of the Inv genes.

We studied sucrose accumulation in Lycopersicon chmielewskii and hexose accumulation in L. esculentum cv. Micro-Tom by using real-time quantitative PCR (RT-PCR) to analyze the expression of Inv genes and invertase inhibitor (INH) at different stages of development and different sites during vegetative and reproductive growth. The essential composition, structure, and expression mechanism of these genes were characterized. This study will provide the foundation for improving the expression and activity of these genes in the plant, changing the rate of sucrose input in the sink, distribution of assimilation products, and improving quality.

\section{MATERIAL AND METHODS}

\section{Plant growth and maintenance}

Seeds (Micro-Tom and L. chmielewskii) were surface-sterilized with $0.5 \% \mathrm{NaOCl}$ and rinsed with water. They were germinated for 3 days in the dark at $25^{\circ} \mathrm{C}$ on 2 layers of sterile filter paper soaked in water, on plastic Petri dishes. Uniformly germinated seedlings were 
transferred to seedling trays containing 1 part peat:1 part perlite: 1 part vermiculite $(\mathrm{v} / \mathrm{v} / \mathrm{v})$, grown in a greenhouse, and planted at the 5-leaf stage.

\section{Tissue collection}

Sampling of the developmental series occurred over a 5-month period and comprised a total of 38 samples. Roots, stems, and leaves were taken in the second, third, fourth, fifth, and sixth euphylla and source leaves. Four fruit developmental stages were defined based on flower opening. The peduncle, sepal, pulp, pectinic, and dissepiments of fruit were gently removed at 22, 33, 44, and 55 days after anthesis. After collection, samples were immediately frozen in liquid $\mathrm{N}_{2}$ and stored at $-80^{\circ} \mathrm{C}$ until RNA extraction.

\section{Extraction of total RNA}

Total RNA was purified from tissue samples using the plant RNAprep Pure kit from Tiangen and on-column DNase I digestion, following manufacturer protocols (Sigma-Aldrich). RNA was quantified by absorbance at $260 \mathrm{~nm}$ and purity was assessed based on absorbance ratios at $260 / 280 \mathrm{~nm}$. The integrity of purified RNA was confirmed by denaturing agarose gel electrophoresis and ethidium bromide staining.

The cDNA samples for real-time RT-PCR were synthesized from $1 \mu \mathrm{g}$ total RNA and random nonamer primers, using the First-Strand Synthesis System (Sigma-Aldrich), then diluted to a final volume of $200 \mu \mathrm{L}$. The amplicons obtained with each primer pair from the cDNA mixture and from a random subset of individual cDNA samples were checked by electrophoresis on $2 \%$ agarose gels and ethidium bromide staining.

\section{Real-time fluorescence quantitative}

Relative quantitative real-time RT-PCR was performed in triplicate with the AB (Applied Biosystems) 7500 Real Time PCR System and the 7500 v2.0.1 software, by the comparative threshold cycle (Ct) method. Actin (accession No. U60480) was chosen as a relative quantitative reference (forward primer: 5'-TGTCCCTATTTACGAGGGTTATGC-3', reverse primer: 5'-AGTTAAATCACGACCAGCAAGAT-3'). Reactions were prepared in a total volume of $20 \mu \mathrm{L}$ containing $2 \mu \mathrm{L}$ template, $1 \mu \mathrm{L} 2 \mu \mathrm{M}$ of each primer, $9 \mu \mathrm{L} 2.5 \mathrm{X}$ Real MasterMix (Roche Applied Science), $5 \mu \mathrm{L}$ sterile water, and $2 \mu \mathrm{L}$ fluorescein as normalization dye. Blank controls were run in triplicate for each master mix. The cycling conditions were as follows: initial denaturation at $95^{\circ} \mathrm{C}$ for 3 min to activate the FastStart Taq DNA polymerase, followed by 40 cycles of denaturation at $95^{\circ} \mathrm{C}$ for $30 \mathrm{~s}$, annealing at $57^{\circ} \mathrm{C}$ for $30 \mathrm{~s}$, and extension at $68^{\circ} \mathrm{C}$ for $1 \mathrm{~min}$. The amplification process was followed by a melting curve analysis, ranging from $60^{\circ}$ to $90^{\circ} \mathrm{C}$, with temperature increasing in $0.2^{\circ} \mathrm{C}$ increments every $10 \mathrm{~s}$. Baseline and $\mathrm{Ct}$ were automatically determined using the $7500 \mathrm{v} 2.0 .1$ software.

The amplification primers were designed using the DNAMAN6.0 software as shown in Table 1. In order to control for genomic DNA contamination, amplification primers were targeted to different exons as described by Expósito-Rodríguez et al. (2008). Information about exon positions in tomato CWI and Inv genes was directly available from databases. Data analysis was performed in OriginPro7.5 and DPS (2000). 


\begin{tabular}{|c|c|c|c|}
\hline$\overline{\text { Gene }}$ & Accession No. & Forward primer $\left(5^{\prime}-3^{\prime}\right)$ & Reverse primer (5'-3') \\
\hline Lin5 & AJ272304 & AAAGGGATCTCAGCATCACAGG & CGTCTTGGGCATATAGGTCAGC \\
\hline Lin6 & AF506005 & ATCAAGCCCGATAACAATCCA & ССТСАСАСТСССАACCAATACTC \\
\hline $\operatorname{Lin} 7$ & AF506006 & TTTGGTGCTGGTGGAAAGACA & GGCTCCGTTCCGTTGTTAAAC \\
\hline Lin8 & AF506007 & AAGGATGGGCGGGAATACA & GGCCTGTGCTGGTGTGATT \\
\hline TIVI & AF465612 & AGGACTTTAGAGACCCGACTAC & GCAGCACTCCATCCAATAGC \\
\hline INH & AJ010943 & GTATGCCAGAAGCATTAGAAGCA & GCATCACCAGAAGAACCAACC \\
\hline
\end{tabular}

\section{RESULTS AND DISCUSSION}

\section{Difference in relative expression of tomato Inv genes and inhibitor during vegetative growth}

Relative quantitative methods of RT-PCR can provide expression levels under various conditions. These methods enhance the accuracy of conventional quantitative analysis in comparison to early quantitative PCR methods, in which products are quantified after electrophoresis with dyes, radioactivity, or probes, followed by manual data collection (Giulietti et al., 2001). Expression of the CWI gene is very low in each stage of growth and development and is almost undetectable by the Northern hybridization method. Lin 5, Lin6, Lin 7, and $\operatorname{Lin} 8$ ESTs were not found in the GenBank database, which also illustrate that the RNA expression level is very low. This study detected expression of CWI by using RT-PCR.

Figure 1 shows that the expression of Lin6 in L. chmielewskii root is higher than in Micro-Tom before the fourth euphylla, after the fifth euphylla has changed; the expression of Lin6 is 2-3 times higher in Micro-Tom than in L. chmielewskii. The expression of L. chmielewskii Lin7 is 6-fold higher than in Micro-Tom; expression of Lin6 was significantly different in the stems with several-fold (up to 10-fold) more in Micro-Tom than in L. chmielewskii. Lin5 expression was observed only in the L. chmielewskii leaves, where Lin 7 was also expressed. The expression of $I N H$ was weak in the roots and stems, but suddenly increased in the fifth euphylla of L. chmielewskii leaves, about 30-fold higher than in Micro-Tom. Lin8 and TIVI were not expressed.

The results show that overall expression of sucrose Inv genes is very low in the vegetative growth stage. Sucrose metabolism does not occur in the "source" tissue, but is transported to the "sink" tissue during vegetative growth. However, changes in the expression of sucrose Inv genes occur in a regular pattern: the expression was lower when the second euphylla appeared, then expression levels elevated when tomato grew up to the fifth euphylla or the sixth euphylla, after which expression levels decreased again. Thus, sucrose is high in young leaves then falls when the leaves increase their function. Related research demonstrated the relationship between high invertase activity and rapid growth of plant tissues (Gayler and Glasziou, 1972). For instance, in the tomato growth zone, invertase activity quickly increased in the cell wall and vacuole, demonstrating its association with plant growth.

Lin6 expression was assayed in cultivated and wild-type plants; its expression was highest of the Inv genes. This finding is consistent with published data (Godt and Roitsch, 1997) and suggests that Lin6 plays a major role in vegetative growth. Moreover, the Lin6 gene 
was induced by glucose, zeatin, brassinosteroid, elicitors, and wounding (Godt and Roitsch, 1997; Tang et al., 1999). Lin6 expression may be regulated by endogenous hormones during vegetative growth.
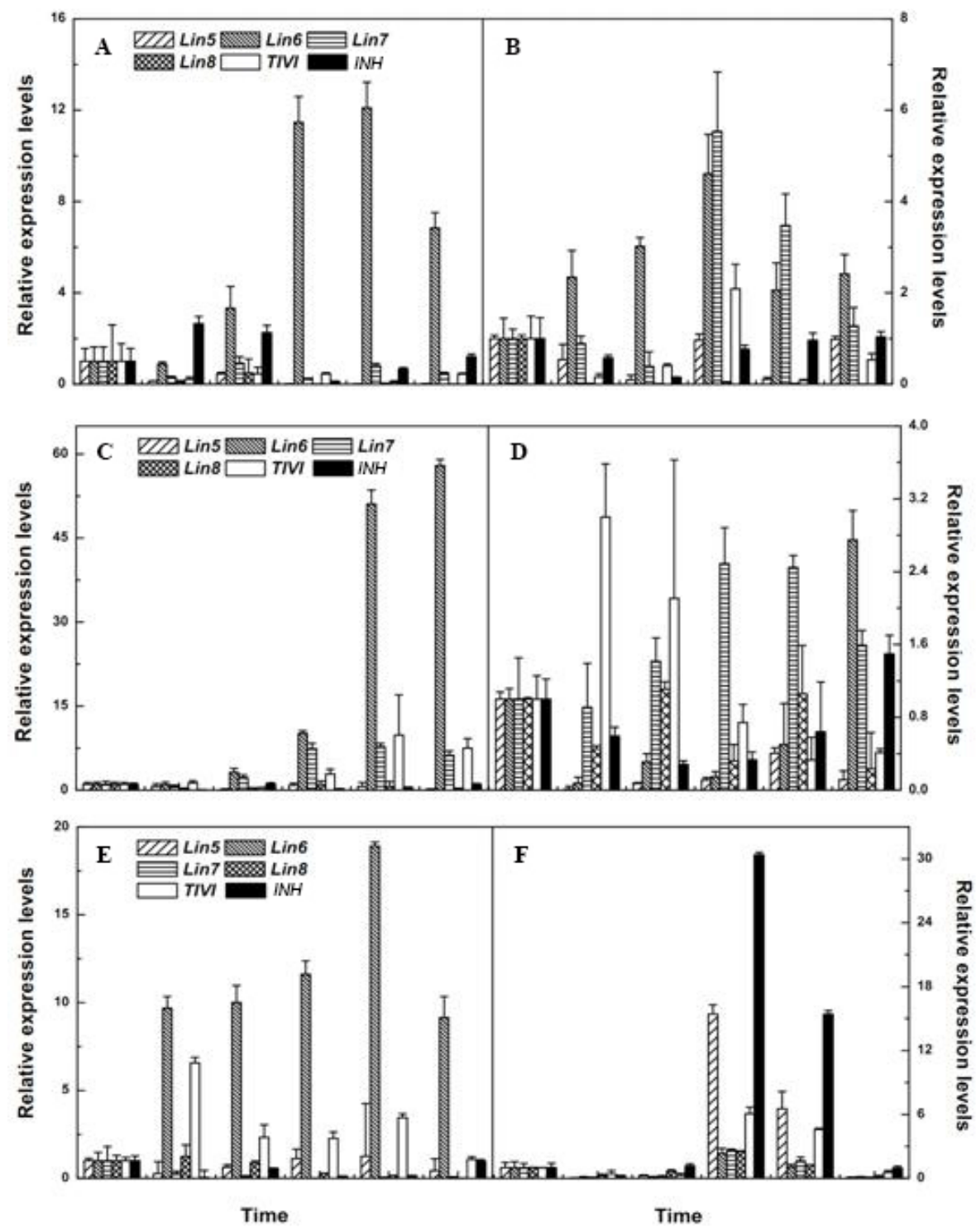

Figure 1. Relative expression levels of tomato sucrose invertase gene families and inhibitor in the vegetative growth stage. The levels of expression were calculated using the standard curve method. The values in the charts resulted from three technical replicates, and by using two independently extracted total RNA samples ( \pm standard error). The expression of sucrose invertase gene families and inhibitor in the roots, stems, and leaves at the second euphylla, third euphylla, fourth euphylla, fifth euphylla, sixth euphylla, and source leaves in Lycopersicon esculentum cv. Micro-Tom (A, C, E) and L. chmielewskii $(\mathbf{B}, \mathbf{D}, \mathbf{F})$. 
The study also found that $\operatorname{Lin} 5$ and $\operatorname{Lin} 7$ were not expressed in the nutritional organs of Micro-Tom, but were expressed in the nutritional organs of $L$. chmielewskii. This is not consistent with previous reports, in which transient expression analysis of Lin 5 promoter sequences fused to a GUS reporter demonstrated specific expression of Lin 5 in tomato flower organs and fruit (Proels et al., 2003). In situ hybridization analysis of $\operatorname{Lin} 7$ revealed high tissue-specific expression in the tapetum and pollen of Nicotiana tabacum (cv. Samsun) and L. esculentum (cv. Micro-Tom). Lin 5 and $\operatorname{Lin} 7$ may be expressed in the nutritional organs of wild-type plants.

Lin 8 was not detected in any of the tissues, meaning Lin 8 is silenced (Godt and Roitsch, 1997). Expression of INH suddenly increased in the fifth euphylla of L. chmielewskii leaves, to levels 30-fold greater than in Micro-Tom. Because of the long growth cycle of the wild species, 5 leaves is still in the early stage of flower bud differentiation. INH can inhibit the sucrose decomposition by inhibiting the expression of Inv gene family, so that the sucrose can be transport and supply for tissue development and flower bud differentiation.

\section{Relative expression levels of tomato sucrose invertases and inhibitor during reproductive growth}

Figure 2 shows that the overall relative expression of sucrose Inv genes was greatly reduced in L. chmielewskii 22 to 55 days after anthesis (DAA); levels were several to hundreds-fold lower than in Micro-Tom and were absent in the dissepiments. Lin6 gene expression was lowest in each part of the Micro-Tom fruit, similar to its expression in L. chmielewskii. Lin8 expression was 20-fold higher than L. chmielewskii at 22 DAA. Lin5, Lin7, and TIVI expression emerged 33 to 44 DAA in Micro-Tom. This also means that 33 to 44 DAA is the key period that determines tomato fruit quality. In addition, the temporally differential expression of the Inv genes was not detected in the peduncle. If relative expression levels of each gene in peduncle at $22 \mathrm{DAA}$ were a reference, the gene expression of both species showed different changes in different periods. These changes indicate that the difference of sucrose accumulation in tomato fruit was mainly due to Inv gene family differentially expressed. Thus, differences in sucrose accumulation in tomato fruit is mainly due to differentially expressed Inv genes in the latter stages of fruit growth.

Overall, relative expression of sucrose Inv genes greatly decreased in L. chmielewskii in the reproductive growth stage, to levels lower than those in Micro-Tom by several- to hundredsfold, and there was no expression in the dissepiments. Sun et al. (1992) studied fruit sucrose metabolism in L. chmielewskii. As a result, the ability to degrade sucrose does not increase in the latter stage of fruit growth and found that, along with fruit growth and development, AI always maintained a low level of activity, leading to sucrose accumulation in the fruit. Our results suggest that the difference in sucrose accumulation in tomato fruit is mainly due to differentially expressed Inv genes during the latter stage of fruit growth. Yelle et al. (1988) also showed that L. chmielewskii accumulated sucrose rather than glucose and fructose, because of sucrose-accumulating trait associated with greatly reduced levels of AI. As it is proposed in plant, sucrose transportation can carry signals to influence gene expression, including the expression of Inv (Gibson, 2005). Furthermore, the expression of Inv is under the control of certain hormones, such as GA, ethylene, and ABA, all of which induce Inv expression (Hein et al., 1984; Wang and Zhang, 2000). However, all reduction on the total Inv gene families remains to be further explored due to sugar, hormone, or control calcium signal. 

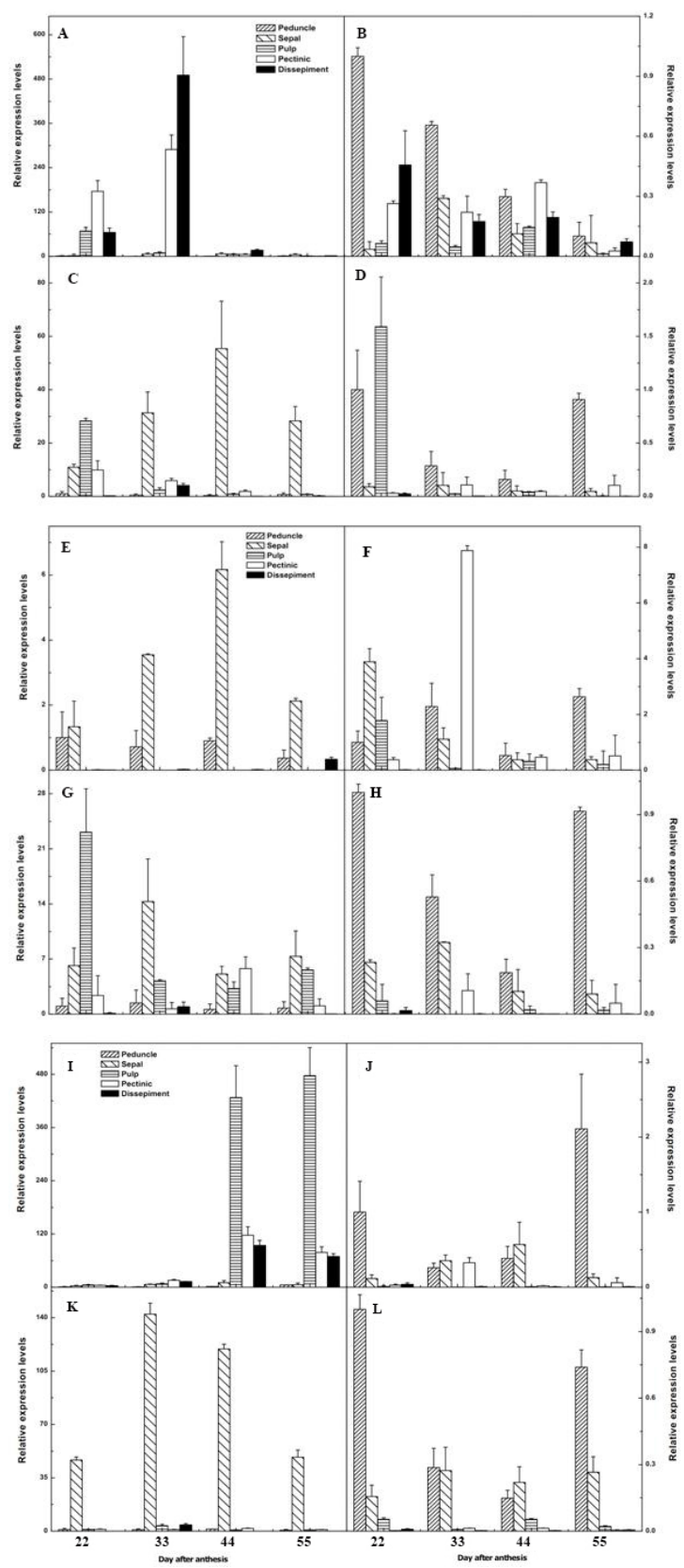

Figure 2. Relative expression levels of tomato sucrose invertase gene families and inhibitor in the reproductive growth stage. The levels of expression were calculated using the standard curve method. The values in the charts resulted from three technical replicates, and by using two independently extracted total RNA samples ( \pm standard error). A. C. E.G. I. K. Relative expression levels of $\operatorname{Lin} 5, \operatorname{Lin} 7, \operatorname{Lin} 6, \operatorname{Lin} 8, T I V I$, and $I N H$ at 22, 33, 44, and 55 days after anthesis in Lycopersicon esculentum cv. Micro-Tom, respectively. B. D. F. H. J. L. Relative expression levels of Lin 5, Lin 7, Lin6, Lin8, TIVI, and INH at 22, 33, 44, and 55 days after anthesis in L. chmielewskii, respectively. 
The cultivated tomato (Micro-Tom) mainly accumulates hexose. Studies have demonstrated that when sucrose invertase activity is high, sucrose accumulation is translated to hexose or hexose phosphate (Qin and Zhang, 2005). Lin6 expression is significantly lower than that of other invertases in each part of the Micro-Tom fruit, similar to its expression in $L$. chmielewskii; the expression of INH in the sepals was dozens of times higher than that of Lin6 in Micro-Tom, which seems to suggest that INH interacts with Lin6. However, colocalization of INH with the cell wall invertase Lin 5 was demonstrated in situ (Jin et al., 2009). This result should be validated by yeast two-hybrid or similar experiments.

Some research shows that the sugar in a tomato fruit enters the vacuoles of pulp cells by facilitated diffusion, but tomato pulp stores most sucrose from the phloem by CWI hydrolysis, as phloem unloading requires a sucrose gradient (Milner et al., 1995). In this study INH expression in the sepal was high, suggesting that high sucrose levels in the sepal induce transport down the sucrose gradient to the fruit, where it nourishes fruit growth and development. The peduncle is not a photosynthetic organ, as its role is more directed toward transportation and support. Inv genes and invertase inhibitor were not detected in the peduncle, suggesting the absence of sucrose catabolism in this organ. The expression of $\operatorname{Lin} 5, \operatorname{Lin} 7$, and TIVI at 33 to 44 DAA in Micro-Tom suggest that this is the key period that determines tomato fruit quality. Invertase activity during tomato fruit maturation is controlled at the transcriptional level (Klann et al., 1993). Our experiment verified this result.

Genotype differences influence the metabolism of sucrose, not its uptake and distribution. The expression of VI in wild tomato is extremely low, as its enzyme activity. Sucrose metabolism is therefore blocked, resulting in sucrose accumulation. This also means that reduced expression of AI is a prerequisite of sucrose storage. Other sucrose-storing plants (such as beet, orange, radish, sugar cane, etc.) also show characteristics consistent with the absence of AI activity (Hatch and Glasziou, 1963; Kato and Kubota, 1978). Cucumber storage of sucrose and hexose also confirms these conclusions (Schaffer et al., 1987). Thus, sucrose storage should occur in the absence of AI. Invertase inhibitor has been reported in sucrose-storing plants such as red carrot, beet, and sweet potato. However, many studies do not support the notion that invertase inhibitor causes loss of AI activity. Immunology experiments can be used to measure the relationship between invertase protein level and activity, to determine whether loss of AI activity is due to reduced or absent enzyme synthesis. This study demonstrated that AI expression is very low during different periods of fruit development and in various parts of wild tomato, and that invertase inhibitor expression is low.

In summary, this study constitutes the first in-depth study to validate and quantify Inv and INH expression during tomato development using RT-PCR technology. We tested the expression of 6 genes in a set of 38 tissue samples from tomato plants. The results of these experiments confirm that extracellular and intracellular invertases are important determinants of carbohydrate metabolism and distribution in tomato sucrose metabolism. High sucrose concentrations were correlated with transcription of Inv genes, indicating the signaling function of sucrose for inducing the transition from "source" to "sink" at the transcriptional level.

\section{ACKNOWLEDGMENTS}

Research supported by grants from the National Natural Science Foundation of China (grant \#30971999); the National Key Basic Research Special Funds of China (grant 
\#2009CB119000); and the Key Laboratory of the Liaoning Province Education Department (grant \#2009S092).

\section{REFERENCES}

Beürter J (1985). Sugar accumulation and changes in the activities of related enzymes during development of the apple fruit. J. Plant Physiol. 121: 331-341.

Expósito-Rodríguez M, Borges AA, Borges-Pérez A and Pérez JA (2008). Selection of internal control genes for quantitative real-time RT-PCR studies during tomato development process. BMC Plant Biol. 8: 131-143.

Gayler KR and Glasziou KT (1972). Sugar accumulation in sugarcane: carrier-mediated active transport of glucose. Plant Physiol. 49: 563-568.

Gibson SI (2005). Control of plant development and gene expression by sugar signaling. Curr. Opin. Plant Biol. 8: 93-102.

Giulietti A, Overbergh L, Valckx D, Decallonne B, et al. (2001). An overview of real-time quantitative PCR: applications to quantify cytokine gene expression. Methods 25: 386-401.

Godt DE and Roitsch T (1997). Regulation and tissue-specific distribution of mRNAs for three extracellular invertase isoenzymes of tomato suggests an important function in establishing and maintaining sink metabolism. Plant Physiol. 115: 273-282.

Hatch MD and Glasziou KT (1963). Sugar accumulation cycle in sugar cane. II. Relationship of invertase activity to sugar content \& growth rate in storage tissue of plants grown in controlled environments. Plant Physiol. 38: 344-348.

Hedley PE, Machray GC, Davies HV, Burch L, et al. (1994). Potato (Solanum tuberosum) invertase-encoding cDNAs and their differential expression. Gene 145: 211-214.

Hein MB, Brenner ML and Brun WA (1984). Concentrations of abscisic acid and indole-3-acetic acid in soybean seeds during development. Plant Physiol. 76: 951-954.

Hubbard NL, Huber SC and Pharr DM (1989). Sucrose phosphate synthase and acid invertase as determinants of sucrose concentration in developing muskmelon (Cucumis melo L.) fruits. Plant Physiol. 91: 1527-1534.

Jiang J and Li TL (2005). Molecular biology research of sucrose metabolism key genes in tomato. Biotechnology 15: $82-85$.

Jin Y, Ni DA and Ruan YL (2009). Posttranslational elevation of cell wall invertase activity by silencing its inhibitor in tomato delays leaf senescence and increases seed weight and fruit hexose level. Plant Cell 21: 2072-2089.

Kato T and Kubota S (1978). Properties of invertases in sugar storage tissues of Citrus fruit and changes in their activities during maturation. Acta Physiol. Plant. 42: 67-72.

Klann EM, Chetelat RT and Bennett AB (1993). Expression of acid invertase gene controls sugar composition in tomato (Lycopersicon) fruit. Plant Physiol. 103: 863-870.

Masao A, Hitoshi M and Hidemasa I (1991). Roles of sucrose-metabolizing enzymes in growth of seedlings. purification of acid invertase from growing hypocotyls of mung bean seedlings. Plant Cell Physiol. 32: 1291-1298.

Milner ID, Ho LC and Hall JL (1995). Properties of proton and sugar transport at the tonoplast of tomato (Lycopersicon esculentum) fruit. Acta Physiol. Plant. 94: 399-410.

Proels RK, Hause B, Berger S and Roitsch T (2003). Novel mode of hormone induction of tandem tomato invertase genes in floral tissues. Plant Mol. Biol. 52: 191-201.

Qin QP and Zhang ML (2005). The research progress of molecular biology about regulation of fruit sugar content and component. J. Fruit Trees 22: 519-525.

Schaffer AA, Aloni B and Fogelman E (1987). Sucrose metabolism and accumulation in developing fruit of cucumis. Phytochemistry 26: 1883-1887.

Schwimmer S, Makower RU and Rorem ES (1961). Invertase \& invertase inhibitor in potato. Plant Physiol. 36: 313-316.

Sun J, Loboda T, Sung SJ and Black CC (1992). Sucrose synthase in wild tomato, Lycopersicon chmielewskii, and tomato fruit sink strength. Plant Physiol. 98: 1163-1169.

Tang GQ, Luscher M and Sturm A (1999). Antisense repression of vacuolar and cell wall invertase in transgenic carrot alters early plant development and sucrose partitioning. Plant Cell 11: 177-189.

Wang YZ and Zhang DP (2000). Regulating effects of ethylene on carbohydrate metabolism in "Starkrimson" apple fruit during the ripening period. Acta Hort. Sin. 27: 391-395.

Yelle S, Hewitt JD, Robinson NL, Damon S, et al. (1988). Sink metabolism in tomato fruit: III. Analysis of carbohydrate assimilation in a wild species. Plant Physiol. 87: 737-740. 